\title{
Estabilidade das variáveis de aptidão física e capacidade funcional de mulheres fisicamente ativas de 50 a 89 anos
}

\author{
Stability of physical fitness and functional capacity \\ in physically active women aged 50 to 89 years
}

\author{
João Pedro da Silva Júnior 1,2 \\ Leonardo José da Silva 1,2 \\ Gerson Ferrari 1 \\ Douglas Roque Andrade 1 \\ Luis Carlos Oliveira 1,2 \\ Mauricio dos Santos 1,2 \\ Victor Keihan Rodrigues Matsudo 1,2
}

1 Centro de Estudos do Laboratório de Aptidão Física de São Caetano do Sul. São Caetano do Sul. SP. Brasil

2 Programa Agita São Paulo. São Paulo, SP. Brasil

Recebido em 01/02/10 Revisado em 14/05/10 Aprovado em 27/07/10
Resumo - O objetivo da pesquisa foi verificar o efeito de um programa de atividade física e estabilidade das variáveis de aptidão física e capacidade funcional de mulheres adultas fisicamente ativas. A amostra foi composta por 34 mulheres entre 50-89 anos, participantes de um grupo de atividade física da Universidade Camilo Castelo Branco. Critérios de inclusão: adesão mínima de 75\% de frequência às aulas (3x/semana) e ter realizado, no mínimo, uma avaliação por ano entre 2005-2007. Todas as avaliações foram realizadas no mês de junho. As medidas antropométricas foram: massa corporal, estatura, circunferência da cintura e quadril; neuromotoras: levantar da cadeira em segundos, levantar da cadeira em 30 segundos, flexão de cotovelo, shuttle-run, equilíbrio estático e dinâmico; e metabólica: marcha estacionária de 2 minutos, foi seguida a padronização CELAFISCS. A análise dos dados foi feita pela ANOVA - One Way seguida do "post hoc de Scheffé", delta percentual e correlação de Spearman Rho. O nível de significância adotado foi o p<0,05. Nas três avaliações realizadas, a massa corporal, índice de massa corporal e relação cintura quadril indicaram valores de excesso de peso. A capacidade funcional demonstrou incremento na força de membros superiores (42\%) e inferiores (5,2\%) e para o equilíbrio $(14,1 \%)$, essas mudanças foram significativas, o mesmo não aconteceu para agilidade $(2,1 \%)$, isso comparando 2005-2007. O índice de massa corporal, relação cintura quadril, força e equilíbrio estático a estabilidade variou de 0,26 a 0,91 ( $\mathrm{p}<0,05)$. O programa de atividade física contribuiu para a manutenção da aptidão física e capacidade funcional de mulheres adultas.

Palavras-chave: Atividade física; Aptidão física; Envelhecimento.

Abstract - The objective of this study was to evaluate the effect of a physical activity program and [on ?] the stability of physical fitness and functional capacity in physically active adult women. Thirty-four adult women aged 50-89 years, who participated in a physical activity group at Camilo Castelo Branco University, were studied. Criteria for inclusion were attendance of at least 75\% of the classes (3x/week) and undergoing at least one assessment per year between 2005 and 2007. All evaluations were conducted in June. Anthropometric (body weight, height, waist-hip ratio), neuromotor (rising from a chair in seconds, rising from a chair in 30 seconds, elbow flexion, shuttle-run, static and dynamic balance), and metabolic parameters (2-min step in place test according to the CELAFISCS standard) were evaluated. One-way ANOVA followed by the post hoc Scheffé test, delta percentage and Spearman's rho correlation were used for data analysis, with the level of significance set at $p<0.05$. In the three evaluations, body weight, body mass index and waist-hip ratio indicated overweight. Analysis of functional capacity showed a significant increase in upper (42\%) and lower (5.2\%) limb strength and in balance (14.1\%) when comparing the results from 2005 to 2007. The same was not observed for agility (2.1\%). For body mass index, waist-hip ratio, strength and static balance, stability ranged from 0.26 to $0.91 \quad(p<0.05)$. The physical activity program contributed to the maintenance of physical fitness and functional capacity in adult women.

Key words: Motor activity; Physical fitness; Aging. 


\section{INTRODUÇÃO}

O envelhecimento é um fenômeno complexo, variável, progressivo e comum a todas as espécies, sendo que envolve mecanismos deletérios que influenciam na habilidade dos indivíduos em desempenhar suas funções básicas ${ }^{1}$. Os mecanismos do envelhecimento não ocorrem necessariamente paralelos à idade cronológica e têm consideráveis variações individuais, levando em consideração a genética, estilo de vida e doenças crônicas que vão interagindo entre si e acabam influenciando a condição em que alcançamos numa determinada idade ${ }^{2}$.

$\mathrm{O}$ crescimento do número de pessoas com maior longevidade não se restringe apenas a países desenvolvidos. No Brasil, segundo dados da pesquisa Tábua Completa de Mortalidade (IBGE) ${ }^{3}$, a expectativa de vida da população brasileira passou de 69,66 anos, em 1998, para 72,86 anos, em 2008.

Hábitos de estilo de vida como alimentação saudável e a prática de atividade física podem minimizar os efeitos deletérios deste processo e garantir ao individuo, durante o processo de envelhecimento, uma vida saudável com autonomia, sendo que esses estão cada vez mais consolidadas na literatura científica ${ }^{4}$.

Visto que o nível de atividade física e da capacidade funcional durante o envelhecimento é extremamente importante e está evidenciado que 95\% a $98 \%$ de idosas fisicamente ativas realizam de forma independente suas atividades básicas e instrumentais da vida diária ${ }^{5}$. Outro fato importante é que indivíduos que conseguem acumular níveis satisfatórios de atividade física semanal consomem menos medicamentos ${ }^{6}$. Os achados evidenciam a importância do incentivo da criação de políticas e programas de atividade física, que poderiam auxiliar na diminuição de custos hospitalares e de medicamentos para o tratamento de doenças associadas ao sedentarismo, na terceira idade.

Doenças e limitações não são consequências inevitáveis do envelhecimento e que o uso de serviços preventivos, eliminação de fatores de risco e adoção de hábitos de vida saudáveis são importantes e determinantes para o envelhecimento saudável ${ }^{9}$. A atividade física é fundamental para a manutenção da independência, e é um importante fator de qualidade de vida. Assim, a manutenção ou incremento dos níveis de força e área muscular em indivíduos idosos pela prática deste tipo de exercício, reforçaria a importância de sua realização quanto à função músculo-esquelética?

Diversos modelos de análises têm sido empregados para analisar o efeito que o volume total de atividade física pode exercer sobre diversas características de saúde dos indivíduos.

Malina ${ }^{8}$, em uma revisão da literatura, analisou a estabilidade dos níveis atividade física e aptidão física em indivíduos de diferentes idades, e sugeriu uma definição para "tracking" como sendo a manutenção da posição relativa dentro de um grupo em relação a um determinado período. Ainda afirmou que, para se estabelecer tais valores, seriam necessárias ao menos duas observações dos mesmos indivíduos, sendo a forma metodológica mais usual, a utilização de correlações de "Pearson" ou "Spearman Rho".

Deste modo, o objetivo deste estudo foi verificar o efeito de um programa de atividade física e estabilidade das variáveis de aptidão física e capacidade funcional de mulheres adultas fisicamente ativas.

\section{PROCEDIMENTOS METODOLÓGICOS}

Este estudo teve como população mulheres com idade igual ou superior a 50 anos que participavam, semanalmente, de atividades físicas do Programa de Inclusão Social e Cidadania (Pisc-Dinda) coordenado pela Universidade Livre da Terceira Idade, da Universidade Camilo Castelo Branco (UNICASTELO) unidade de São Paulo. O Programa atendia por volta de 150 participantes/ano.

A amostra do presente estudo foi composta por 34 mulheres adultas, participantes do programa de atividade física, com idades entre 50 a 89 anos $(65,0 \pm 7,19$ anos) e que atendiam os seguintes critérios de inclusão: a) ser do sexo feminino; b) não participar de nenhum outro programa de atividade física; c) adesão mínima de $75 \%$ das aulas; d) ter realizado no mínimo três avaliações consecutivas e completas entre 2005 e 2007.

O programa continha monitoramento semestral das variáveis de aptidão física, capacidade funcional, avaliação cognitiva e psicológica, sendo levado em consideração a coleta de dados do mês de junho, que foi realizada nas dependências da UNICASTELO, por uma equipe previamente treinada, composta por acadêmicos e profissionais de Educação Física da UNICASTELO. O protocolo de atividade física foi composto por aulas de fortalecimento muscular localizado, flexibilidade, alongamento, ritmo, lateralidade, jogos adaptados e aulas de hidroginástica, realizadas três dias por semana, com duração de 60 minutos cada sessão, com intensidade moderada mediante a percepção subjetiva de esforço ${ }^{4}$.

Antes do início das avaliações, toda a amostra e seus respectivos responsáveis preencheram um termo de consentimento, autorizando o uso dos 
seus dados. Nesse termo, constava que não haveria identificação nominal e que poderiam abandonar os testes em qualquer momento se desejassem. Esta pesquisa foi aprovada pelo Comitê de Ética em Pesquisa da Universidade Camilo Castelo Branco, sob o número 1296-1626/06.

Foram mensuradas as variáveis de aptidão física: a) antropométricas: massa corporal (kg), estatura $(\mathrm{cm})$ e calculado o índice de massa corporal (IMC; $\left.\mathrm{kg} / \mathrm{m}^{2}\right)$, circunferência de cintura $(\mathrm{cm})$ e quadril $(\mathrm{cm})$, posteriormente realizado cálculo da relação cintura-quadril (RCQ); b) neuromotoras: força de membros inferiores utilizando os testes de levantar da cadeira (MMIIa; seg), levantar da cadeira em 30 segundos (MMII ${ }^{\text {b }}$ rep), força de membros superiores (MMSS) mediante o teste de flexão de cotovelo (rep), agilidade corporal pelo teste "shuttle-run" (seg), locomoção por meio da velocidade de andar (locomoção) e velocidade máxima de andar (locomoção Max) (seg) e equilíbrio estático utilizando-se o teste flamingo (seg); c) potência aeróbica foi mensurada segundo o teste de marcha estacionária de 2 minutos (rep). Todos os testes e medidas foram aplicados por avaliadores previamente treinados e seguindo o protocolo sugerido por Matsudo9. Os valores de reprodutibilidade das variáveis antropométricas variaram de 0,91 a 0,99 . Já as variáveis neuromotoras se modificaram de 0,48 a 0,92 e metabólica teve um valor de 0,74.

Para verificar a distribuição dos dados, foi utilizado o teste de "skiwness" e a análise descritiva foi utilizada para caracterização da amostra através da média $(\bar{X})$ e desvio-padrão (s). Para comparação dos anos, foi feita a análise de Variância "One Way", seguida de "post hoc de Scheffé" para localizar as possíveis diferenças entre os três anos de avaliação dos valores brutos e a variação percentual entre os anos foi avaliada mediante o delta percentual $(\Delta \%)$ para expressar as mudanças em percentual entre os anos de 2005 e 2007. Para identificar a estabilidade das variáveis, foi realizada a correlação de "Spearman Rho" e levou-se em consideração a classificação proposta por Malina ${ }^{10}$, sendo baixo para $\leq 0,30$, moderado de 0,31 a 0,60 e alto para valores $>0,61$. $\bigcirc$ nível de significância adotado foi de $\mathrm{p}<0,05$.

Os cálculos foram realizados pelo software "Statistical Package for the Social Sciences (SPSS)" versão "14.0 for Windows".

\section{RESULTADOS}

Os valores descritos em média e desvio padrão mostram que as variáveis antropométricas, durante o processo de envelhecimento para este grupo, não apresentaram diferenças estatisticamente significativas entre os anos de 2005 e 2007. Analisando as modificações de 2005 para 2007, o delta percentual apresenta uma manutenção da massa corporal e da estatura e uma diminuição para IMC e RCQ (Tabela 1).

Analisando as variáveis de aptidão física, nota-se que a força de membros inferiores (MMII ${ }^{a}$ ) teve uma melhora de $8 \%$, uma diminuição significativa de 2005 para 2007 e 2006 para 2007. Já a força de membros superiores apresentou diferença significativa ( $p<0,05)$, atingindo uma evolução de $42 \%$ entre os anos de 2005 e 2007. Já força de membros inferiores $\left(\mathrm{MMII}^{\mathrm{b}}\right)$ não apresentou mudanças significativas, assim como a agilidade (Tabela 2 e Figura 1).

Tabela 1. Características antropométricas e as mudanças percentuais entre 2005 - 2007 de mulheres adultas fisicamente ativas no período de três anos.

\begin{tabular}{lccccccc}
\hline & \multicolumn{2}{c}{2005} & \multicolumn{2}{c}{2006} & & 2007 & \multicolumn{2}{c}{$2005-2007$} \\
\hline Variáveis & $\bar{X}$ & $\mathrm{~s}$ & $\bar{X}$ & $\mathrm{~s}$ & $\bar{X}$ & $\mathrm{~s}$ & $\Delta \%$ \\
Massa corporal & 68,2 & 16,5 & 68,6 & 16,4 & 68,3 & 15,9 & 0,14 \\
Estatura & 152,0 & 7,9 & 152,1 & 8,2 & 152,0 & 8,2 & 0,00 \\
IMC & 28,7 & 5,0 & 28,7 & 4,1 & 28,6 & 5,2 & $-0,30$ \\
RCQ & 0,91 & 0,1 & 0,99 & 0,1 & 0,89 & 0,7 & $-2,19$ \\
\hline
\end{tabular}

$\mathrm{p}<0,05-{ }^{\mathrm{a}} 2005 \times 2006 ;{ }^{b} 2005 \times 2007 \mathrm{e}^{\mathrm{c}} 2006 \times 2007$.

Tabela 2. Valores das variáveis de aptidão física de mulheres adultas fisicamente ativas no período de três anos.

\begin{tabular}{|c|c|c|c|c|c|c|c|}
\hline \multirow[b]{2}{*}{ Variáveis } & \multicolumn{2}{|c|}{2005} & \multicolumn{2}{|c|}{2006} & \multicolumn{2}{|c|}{2007} & \multirow{2}{*}{$\frac{2005-2007}{\Delta \%}$} \\
\hline & $\bar{X}$ & $\mathrm{~s}$ & $\bar{X}$ & $\mathrm{~s}$ & $\bar{X}$ & $\mathrm{~s}$ & \\
\hline Força de MMII & $0,62^{a b}$ & 0,1 & 0,62 & 0,1 & 0,57 & 0,1 & $-8,0$ \\
\hline Força de MMSS & $18,6^{b}$ & 3,6 & 19,7 & 3,6 & 26,5 & 6,7 & 42,4 \\
\hline Agilidade & 0,91 & 0,1 & 0,99 & 0,1 & 0,98 & 0,7 & 2,1 \\
\hline
\end{tabular}

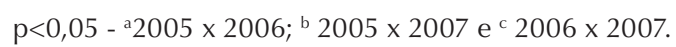




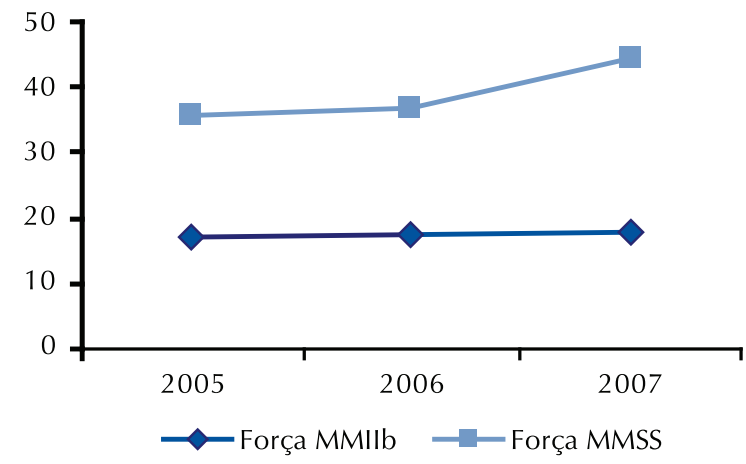

Figura 1. Estabilidade das variáveis de aptidão física de mulheres adultas fisicamente ativas no período de três anos.

Analisando a Tabela 3, a variável equilíbrio estático obteve uma melhora significativa de 14,1\%, tendo diferenças de 2005 para 2007 e 2006 para 2007. A locomoção mostrou um decréscimo de $11,5 \%$, de 2005 para 2007, sendo significativa para esses respectivos anos. Não foi encontrada nenhuma diferença significativa tanto para locomoção máxima quanto para potência aeróbica.

Quando analisamos os valores de correlação, com o propósito de identificarmos a estabilidade (tracking) das principais variáveis de aptidão física e funcional do grupo de mulheres fisicamente ativas, encontramos associações, variando de moderadas a altas e estatisticamente significantes em todas as variáveis (Tabela 4), com exceção para locomoção e locomoção máxima.

Analisando a estabilidade dos achados do presente estudo, tanto a variável IMC quanto RCQ obtiveram valores elevados e estatisticamente significativos $(p<0,05)$. O resultado da força de membros inferiores encontrado foi de 0,60 , estatisticamente significativo $(\mathrm{p}<0,05)$. Para variável força dos membros superiores, foi encontrada uma estabilidade baixa, porém estatisticamente significativa $(0,26 ; p<0,05)$, ainda, uma diferença de $42 \%$ quando comparados os anos de 2005 e 2007, sendo o menor valor de estabilidade encontrado.
Tabela 4. Valores de estabilidade das variáveis de aptidão física e capacidade funcional de mulheres fisicamente ativas.

\begin{tabular}{|c|c|}
\hline Variável & Rho \\
\hline IMC & $0,91^{*}$ \\
\hline RCQ & $0,50^{*}$ \\
\hline Força MMII ${ }^{b}$ & $0,60^{*}$ \\
\hline Força MMSS & $0,26^{*}$ \\
\hline Equilíbrio Estático & $0,42^{*}$ \\
\hline $\mathrm{MMII}^{\mathrm{a}}$ & $0,72^{*}$ \\
\hline Locomoção & 0,11 \\
\hline Locomoção Max & $-0,51$ \\
\hline
\end{tabular}

${ }^{*} \mathrm{p}<0,05$

\section{DISCUSSÃO}

Os resultados demonstram que o programa de atividade física promoveu impacto positivo nas variáveis analisadas pelo presente estudo. Esse fato demonstra que, mesmo as mulheres envelhecendo, ainda é possível a manutenção da aptidão física e capacidade funcional, facilitando as atividades da vida diária.

Matsudo et al. ${ }^{10}$ encontraram resultados semelhantes (Tabela 1) em relação às variáveis antropométricas, isso quando submeteram 117 idosas (50-79 anos) a um programa de atividade física sistematizado. As variáveis antropométricas não sofreram grandes alterações mediante o impacto da atividade física que estaria associado na manutenção dos níveis dessas variáveis. Provavelmente, o estímulo para reduzir a composição corporal não foi suficiente nesses protocolos aos quais essas amostras foram submetidas.

A média do IMC encontrado neste estudo classificou o grupo de mulheres praticantes de atividade física como excesso de peso (OMS $)^{11}$. Resultados semelhantes foram achados por diversos outros estudos na literatura ${ }^{12,13}$. Tanto Gomes et $\mathrm{a}^{12}$ quanto Pauli et al. ${ }^{13}$ verificaram que mulheres idosas fisicamente ativas, participantes de um programa de atividade física tinham valores elevados de IMC,

Tabela 3. Valores das variáveis de capacidade funcional de mulheres adultas fisicamente ativas no período de três anos.

\begin{tabular}{lccccccc}
\hline & \multicolumn{2}{c}{2005} & \multicolumn{2}{c}{2006} & \multicolumn{2}{c}{2007} & $2005-2007$ \\
\hline Variáveis & $\bar{X}$ & $\mathrm{~s}$ & $\bar{X}$ & $\mathrm{~s}$ & $\bar{X}$ & $\mathrm{~s}$ & $\Delta \%$ \\
Equilíbrio & $23,3^{\mathrm{b}}$ & 4,6 & $22,9^{\mathrm{c}}$ & 4,7 & 26,6 & 4,9 & 14,1 \\
Locomoção & $2,67^{\mathrm{b}}$ & 0,34 & 2,69 & 0,31 & 2,94 & 0,58 & 11,5 \\
Locomoção Max. & 2,28 & 0,8 & 2,29 & 0,10 & 2,42 & 0,34 & 6,14 \\
Potência Aeróbica & 86,3 & 9,3 & 90,5 & 11,3 & 87,2 & 14,3 & 0,3 \\
\hline
\end{tabular}

$\mathrm{p}<0,05$ - a $2005 \times 2006 ;{ }^{\text {b }} 2005 \times 2007$ e ${ }^{\text {c } 2006} \times 2007$. 
sendo que esse último foi visto após 12 anos de prática de atividade física sistematizada. Em sua pesquisa, Mazo et a ${ }^{14}$ concluem que há uma relação inversa entre o índice de aptidão física geral com variáveis antropométricas como massa corporal e IMC, ou seja, níveis mais altos de IMC estão relacionados ao desempenho mais baixo em testes de aptidão funcional em mulheres idosas. Contudo, exibir um IMC que represente excesso de peso pode não ser associado a maior taxa de mortalidade por todas as causas entre pessoas acima de 65 anos $^{15}$. No entanto, o estímulo em manter o peso corporal dentro dos limites desejáveis deve ser encorajado a toda pessoa idosa, a fim de reduzir a incidência de outras doenças como o diabetes, por exemplo.

Concordando com os nossos achados, Rogato et al. ${ }^{16} \mathrm{e}$ Gomes et al. ${ }^{12}$ encontraram que as mulheres praticantes de atividades físicas tinham alto indicador de risco para desenvolvimento de doenças cardiovasculares identificado pela alta relação cintura quadril. Dados estes que são corroborados com outros estudos da literatura que já evidenciaram que a distribuição de gordura corporal possui maior acúmulo na região abdominal visceral com o avanço da idade, mesmo entre indivíduos com envolvimento regular em programas de atividade física supervisionada $a^{17,18}$.

Outros autores ${ }^{13,19}$ também evidenciaram que a prática de atividade física e orientação nutricional para mulheres idosas fisicamente ativas contribuem para um menor valor de massa corporal, IMC, porcentagem de gordura corporal e relação cintura quadril, quando comparadas com indivíduos idosos sedentários e fisicamente ativos. Este fenômeno pode ser explicado por alterações no balanço energético, ao longo dos anos em função do incremento do nível de atividade física e melhor consumo alimentar. Em estudo anterior, analisado por um período de quatro anos, Matsudo et al ${ }^{20}$ encontraram valores antropométricos similares aos do nosso estudo, também em mulheres fisicamente ativas.

Em relação à potência muscular, o resultado do presente estudo mostrou uma redução de $8 \%$ da potência dos membros inferiores das mulheres. Em contrapartida, Lovell et al. ${ }^{21}$ verificaram que um programa de treinamento realizado três vezes por semana de intensidade moderada a alta resultou em melhoras significativa da força $(21 \%)$ e da potência (10\%) muscular, no entanto, esse ganho foi perdido após 4 semanas de destreinamento. Mais uma vez, o estímulo a que as mulheres foram submetidas não foi suficiente para alterar de forma positiva a potência muscular. Outro possível mecanismo que explicaria essa redução foi encontrado por Andrade et $a{ }^{22}$ que descrevem que a força muscular decresce com a idade, tendo o seu comprometimento em membros inferiores mais intenso do que em membros superiores. Os resultados do presente estudo mostraram, ainda, que mulheres fisicamente ativas, quando submetidas a um programa de atividade física obtiveram uma melhora significativa de $42,4 \%$ na força de membros superiores e 5,2\% para os membros inferiores, porém não significativa. Já Silva et al. ${ }^{23}$ submeteram 30 idosas a um programa de treinamento com pesos e notaram que a força dos membros inferiores aumentou em 35\% e 15\% para os membros inferiores, ambos significativos. Porém, pesquisa anterior já havia encontrado aumento de 174\% na força de membros inferiores quando submetidos a uma intensidade de $80 \%$ de 1RM. Nota-se, então, que a resposta ao treinamento está diretamente relacionada à carga a que são submetidos ${ }^{24}$.

Fronteira et al. ${ }^{25}$, ao acompanhar um estudo longitudinal durante dez anos, evidenciaram que a prática de atividade física foi associada à preservação da massa magra e diminuição da força, que ocorre de forma diversificada e não de forma homogênea e que a atividade física minimiza os efeitos deletérios inevitáveis relacionados à sarcopenia que ocorre durante o processo de envelhecimento ${ }^{25}$.

Em relação à capacidade funcional mostrada na Tabela 3, Benedetti e Petroski ${ }^{26}$ encontraram dados similares, analisando os efeitos de um programa de atividade física em mulheres institucionalizadas entre 59 a 73 anos de idade. Os autores observaram melhora significativa no equilíbrio estático e flexibilidade em um período de cinco meses de intervenção. Já Pauli et al. ${ }^{13}$ verificaram, em um estudo longitudinal de 12 anos, que as idosas participantes de um programa de atividade física regular tiveram os valores de flexibilidade mantidos no mesmo nível e a agilidade, juntamente com o equilíbrio dinâmico, obtiveram melhoras significativas nas avaliações pré e pós.

Dados interessantes foram publicados por Manini et al..$^{27}$ que analisaram grupos de idosos com diferentes níveis de atividade física, evidenciando que limitações de mobilidade estavam associadas com baixos níveis de atividade física, chegando a encontrar diferenças de $40 \%$ entre os grupos de indivíduos ativos e sedentários.

Mudanças no nível de atividade física e seu impacto nos aspectos ligados à saúde têm recebido grande atenção por parte de pesquisadores da área, Byberg et al. ${ }^{28}$ encontraram um aumento da incidência de mortalidade para todas as causas em 
idosos de acordo com a diminuição dos níveis de atividade física. Já Ramos ${ }^{29}$, analisando uma coorte de idosos residentes em centro urbano, concluiu que a perda da capacidade funcional estava associada ao maior risco de mortalidade para todas as causas. Cabe ressaltar, no entanto, que aos 50 anos de idade a expectativa de vida pode alcançar 3,5 anos para mulheres e 3,7 anos para os homens, desde que mantenham-se ativos, independentemente da presença ou não de doenças cardiovasculares ${ }^{30}$.

Em relação aos dados de estabilidade, Matsudo et al. ${ }^{20}$ realizaram um estudo longitudinal de 4 anos com mulheres brasileiras fisicamente ativas, sendo encontrado valor de correlação de 0,42 para membros inferiores e de 0,61 para membros superiores, ambos significativos. Os resultados foram similares ao do presente estudo. Nota-se que os achados do nosso estudo para força de membros inferiores foram maiores e para força dos membros superiores os resultados foram menores.

Nelson et al. ${ }^{4}$ citam que os idosos que adquirem aumento na força muscular possuem diversos benefícios: diminuição nas quedas que são muito frequentes em indivíduos de idade avançada, melhora do tempo de reação, sinergia motora das reações posturais, velocidade no andar, mobilidade e flexibilidade.

Assim, podemos identificar que uma estratégia eficaz, visando o impacto positivo nas variáveis de aptidão física e capacidade funcional, poderia ser atribuída à participação de indivíduos idosos em programas de atividades físicas estruturadas ou até mesmo em atividades utilitaristas como a caminhada no lazer ou em caráter de transporte.

Apesar da riqueza de um delineamento longitudinal, o estudo apresenta algumas limitações como não ter analisado a amostra por década etária, já que o tamanho da mesma não permitiu a respectiva análise. Outro fato a ressaltar é que um tempo maior de análise possibilitaria uma menor influência do treinamento, e representaria melhor a estabilidade das variáveis.

\section{CONCLUSÃO}

A participação de idosas em programas supervisionados de atividade física promove efeito positivo na manutenção dos níveis nas variáveis antropométricas. Já o efeito do programa de atividade física para as variáveis de capacidade funcional: força de membros superiores, inferiores e equilíbrio estático foram encontradas diferenças positivas e significativas quando comparados os anos 2005 e 2007, tendo apenas a locomoção e locomoção máxima apresentando diminuição de desempenho. Foi observado, ainda, grande variabilidade na estabilidade das variáveis de aptidão física e funcional durante o processo de envelhecimento dessas senhoras.

Estes dados reforçam que um programa de atividade física ajuda na manutenção da aptidão física e na capacidade funcional em idosas. Tal fato apoia a necessidade do estímulo da atividade física regular após os 50 anos de idade.

\section{REFERÊNCIAS BIBLIOGRÁFICAS}

1. Heikkinen RL. The role of physical activity in healthy aging. Geneva, World Health Organization; 1998.

2. Mazzeo RS, Cavanagh P, Evans WJ, Fiatarone MA, Hagberg J, McAuley E, et al. Exercício e atividade física para pessoas idosas. Posicionamento oficial do American College of Sports Medicine. Rev Bras Ativ Fis Saúde 1998;3(1):48-78

3. Instituto Brasileiro de Geografia Estatística/IBGE. Disponível em: http://www.ibge.gov.br/home/presidencia/ noticias/noticia_visualiza.php?id_noticia=1275\&id_pagina=1 [ 2009 nov 20].

4. Nelson ME, Rejeski WJ, Blair SN, Blair SN, Duncan PW, Dudge JO, et al. Physical activity and public health in older adults: Recommendation from the American College of Sports Medicine and the American Heart Association. Circulation 2007;116(9):1094-105.

5. Matsudo SM, Matsudo VK, Barros TL. Efeitos benéfi$\cos$ da atividade física na aptidão física e saúde mental durante o processo de envelhecimento. Rev Bras Ativ Fis Saúde 2000; 5(3):60-76.

6. Willians PT. Reduced diabetic, hypertensive and cholesterol medication use with walking. Med Sci Sports Exerc 2008:40(3):433-43.

7. Matsudo SMM. Evolução da aptidão física e capacidade funcional de mulheres ativas acima de 50 anos de idade de acordo com a idade cronológica. [Tese de Doutorado]. São Paulo (SP): Universidade Federal de São Paulo; Escola Paulista de Medicina; 2001.

8. Malina RM. Tracking of physical activity and physical fitness across the lifespan. Res Q Exerc Sport 1996;67:48-57.

9. Matsudo SMM. Avaliação do idoso - física \& funcional. $2^{a}$ edição. Revisada e atualizada. Londrina, Midiograf, 2005.

10. Matsudo SMM, Barros TL, Matsudo VK. Perfil Antropométrico de mulheres maiores de 50 anos fisicamente ativas, de acordo com a idade cronológica - evolução de 1 ano. Rev Bras Ciênc Mov 2002;10(2):15-26.

11. World Health Organization. Obesity: Preventing and managing the global epidemic. Report of a WHO consultation. World Health Organ Tech Rep Ser. 2000; 894:1-253.

12. Gomes MA, Rech CR, Gomes MBA, Santos DL. Correlação entre indices antropométricos e distribuição de gordura corporal em mulheres idosas. Rev Bras 
Cineantropom Desempenho Hum 2006;8(3):16-22.

13. Pauli JR, Souza LS, Zago AS, Gobbi S. influência de 12 anos de prática de atividade física regular em programa supervisionado para idosos. Rev Bras Cineantropom Desempenho Hum 2009;11(3):255-60.

14. Mazo GZ, Külkamp W, Lyra VB, Prado APM. Aptidão functional geral e índice de massa corporal de idosas praticantes de atividade física. Rev Bras Cineantropom Desempenho Hum 2006;8(4):46-51.

15. Kuk JL; Ardern CI. Influence of Age on the Association Between Various Measures of Obesity and All-Cause Mortality. J Am Geriatr Soc 2009;57:2077-84.

16. Rogatto GP, Gobbi S. Efeitos da Atividade Física Regular Sobre Parâmetros Antropométricos e Funcionais de Mulheres Jovens e Idosas. Rev Bras Cineantropom Desempenho Hum 2001;3(1):63-9.

17. Huang B, Rodriguez BL, Burchfiel CM, Chyou P, Curb JD, Sharp DS. Associations of adiposity whith prevalent coronary heart disease among elderly men: the Honolulu health program. Int J Obesity 1997;21:340-8.

18. Krause MP, Buzzachera CF, Hallage T, Santos ECR, Silva SG. Alterações morfológicas relacionadas à idade em mulheres idosas. Rev Bras Cineantropom Desempenho Hum 2006;8(2):73-7.

19. Ferreira MT, Matsudo SMM, Matsudo VKR, Braggion. Efeitos de um programa de intervenção de atividade física e orientação nutricionais em senhoras fisicamente ativas acima de 50 anos de idade. Rev Bras Med Esporte 2005;11(3):172-6

20. Matsudo SMM, Marin RV, Ferreira MT, Arauújo TL, Matsudo VKR. Estudo longitudinal-tracking de 4 anosda aptidão física de mulheres da maioridade fisicamente ativas. Rev Bras Ciênc Mov 2004;12(3):47-52.

21. Lovell DI, Cuneo R, Gass GC. Can Aerobic training improve muscle strength and power in older men? J Aging Phys Act 2010;18:14-26.

22. Andrade EL, Matsudo SMM, Matsudo VKR. Performance neuromotora em mulheres ativas. Rev Bras Ativ Fís Saúde. Londrina. 1995;1(2):5-15.

23. Silva CM, Gurjão ALM, Ferreira L, Gobbi LTB, Gobbi S. Efeito do treinamento com pesos, prescrito por zona de repetições máximas, na força muscular e composição corporal em idosas. Rev Bras Cineantropom Desempenho Hum 2006;8(4):39-45.
24. Fiatarone MA, Marks EC, Ryan ND, Meredith CN, Lipsitz LA, Evans WJ. High-intensity strength training in nonagenarians. Effects on skeletal muscle. J Am Med Assoc 1990;263:3029-34.

25. Fronteira WR, Reid KF, Phillips EM, Krivickas LS, Hughes VA, Roubenoff R, et al. Muscle fiber size and function in elderly humans: a longitudinal study. J Appl Physiol 2008; 105: 637-42.

26. Benedetti T, Petroski E. Idosos asilados e pratica de atividade física. Rev Bras Ativ fis Saúde 1999;3(2):5-16.

27. Manini TM, Everhart JE, Patel KV, Schoeller DA, Cummings S, Mackey DC, et al. Activity energy expeniture and mobility limitation in older adults: Differential associations by sex. Am J Epidemiol 2009;169(12):1507-16.

28. Byberg L, Melhus H, Gedeborg R, Sundström J, Ahlbom A, Zethelius B, et al. Total mortality after changes in leisure time physical activity in 50 year old men: 35 year follow-up of population based cohort (abstract). Br Med J 2009;338:b688.

29. Ramos LR. Fatores determinantes do envelhecimento saudável em idosos residentes em centro urbano: Projeto Epidoso, São Paulo. Cad Saúde Pública 2003: 19(3):793-8.

30. Franco OH, De Laet C, Peeters A, Jonker J, Mackenbach J, Nusselder W. Effects of physical activity on life expectancy with cardiovascular disease. Arch Intern Med 2005;165:2355-60.

\section{Endereço para correspondência}

João Pedro da Silva Júnior

Rua Heloísa Pamplona, 269 - sala 31.

Bairro Fundação

CEP 09520-320. São Caetano do Sul. SP. Brasil.

E-mail: junior@agitasp.org.br, celafiscs@celafiscs.org.br 